\title{
Evaluation of UHRF1 and P16INK4A expression levels in newly diagnosed AML patients
}

\author{
Vahid Amiri ${ }^{1}$, Mohamadhossein Mohammadi ${ }^{1,2}$, Mohammad Reza Khosravi Farsani ${ }^{3}$, Arshia Gharehbaghian ${ }^{4}$, \\ Abbas Hajifathali ${ }^{2}$, Zaher Khazaei ${ }^{5}$, Mehdi Allahbakhshian Farsani ${ }^{1,2, *}$
}

\begin{abstract}
${ }^{1}$ Department of Laboratory Hematology and Blood Bank, School of Allied Medical Sciences, Shahid Beheshti University of Medical Sciences, Tehran, Iran
\end{abstract}

${ }^{2}$ HSCT Research Center, Shahid Beheshti University of Medical Science, Tehran, Iran

${ }^{3}$ Assistant professor of Medical Hematology-Oncology, Hematology -Oncology Department, Azahra Hospital, Isfahan University of Medical Sciences, Isfahan, Iran

${ }^{4}$ School of Biology, University of Tehran, Tehran, Iran

${ }^{5}$ Cellular and Molecular Research Center, Sabzevar University of Medical Sciences, Sabzevar, Iran

\section{Correspondence}

Mehdi Allahbakhshian Farsani, Department of Laboratory Hematology and Blood Bank, School of Allied Medical Sciences, Shahid Beheshti University of Medical Sciences, Tehran, Iran

HSCT Research Center, Shahid Beheshti University of Medical Science, Tehran, Iran

Email: Allahbakhshian@sbmu.ac.ir History

- Received: 8 May 2018

- Accepted: 13 August 2018

- Published: 27 September 2018

DOI : 10.15419/bmrat.v5i9.475

\section{Check for updates}

\section{Copyright}

(c) Biomedpress. This is an openaccess article distributed under the terms of the Creative Commons Attribution 4.0 International license.

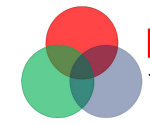

BioMedPress The Open Access Publisher

\begin{abstract}
Introduction: Gene mutation is an infrequent cause of tumor suppressor gene (TSG) defect in de novo AML patients. Instead, it seems that leukemic cells employ epigenetic tricks to attenuate the negative impacts of intact TSGs. Ordinarily, critical TSGs, such as p 16INK4A, is hyper-methylated in AML blasts under the impact of master epigenetic regulators, such as UHRF1. In this study, we investigated the correlation between UHRF1 and p16INK4A gene expression levels in newly diagnosed AML patients. Methods: Bone marrow and peripheral blood samples were obtained from 50 newly diagnosed AML patients and 18 healthy normal control subjects. Gene expression levels of UHRF1 and P16INK4A were surveyed using SYBR Green Quantitative Real-time PCR. Statistical analyses were done using SPSS statistical software 21.0. Results: P16INK4A gene expression showed reduced levels in $80.64 \%$ of patients above 45 years of age, while only $32 \%$ of patients below 45 years had reduced expression levels. The Spearman correlation test also demonstrated a significant negative correlation between UHRF1 and p16INK4A gene expression levels in AML patients, which was not observed in the control group $(r=0.343$ and $P=0.015)$. Conclusion: Regarding the age-related patterns of UHRF1 and p16INK4A gene expression, and also the presence of negative correlation between them, we conclude that UHRF1 may potentially be involved in p16INK4A down-regulation in elderly $A M L$ patients, which may subsequently facilitate the progression of $A M L$ in older ages.

Key words: Acute myelogenous leukemia, Gene expression, p16INK4A, UHRF1
\end{abstract}

\section{INTRODUCTION}

Acute myelogenous leukemia (AML) is a clonal malignant disease of hematopoietic system, which accumulates in bone marrow and disrupts normal hematopoiesis ${ }^{1}$. Despite increased understanding of AML pathophysiology, the mortality rate of $\mathrm{AML}$ patients is relatively higher than other hematologic malignancies ${ }^{2}$. Besides the disadvantages of current treatment protocols, one reason for poor outcome in AML patients is the absence of an entirely accurate prognostic marker in patients with normal karyotype (which includes nearly $50 \%$ of AML patients) ${ }^{3}$. Therefore, new studies are necessary for the determination of appropriate prognostic markers and effective treatment protocols. In this regard, recent studies have implicated that epigenetic modifications play a causal role in the initiation and progression of cancer at least as frequently as mutation ${ }^{4,5}$.

Some of the evidence have suggested the prominent pathogenic role of epigenetics in the aggressive behavior of AML versus other genetic lesions ${ }^{6}$. For instance, when compared with other human cancers, AML has a paucity of genetic lesions per case ${ }^{7}$. Also, recent studies have shown that many AML patients do not carry any recognized AML-associated driver gene mutations ${ }^{3}$. Finally, in contrast to solid tumors, sequencing results have shown an infrequent rate of mutations in TSGs in AML patients (except for mutations in WT1 and p53 genes which occur in 10\% and $7 \%$ of AML patients, respectively) ${ }^{8}$.

In comparison with solid tumors, leukemic myeloblasts mainly undergo genome-wide hypermethylation ${ }^{9}$, which represses mainly gene expression of $\mathrm{TSGs}^{10}$. On the other hand, epigenetic modifications in AML patients have a tendency to induce new mutations ${ }^{10}$. The expression of some TSGs is an age-dependent process and increases with aging, probably to prevent clonal expansion of cells that have sufficient number of tumorigenic mutations ${ }^{11,12}$. In this regard, we studied the expression of p16INK4A, a tumor preventive gene, as it has this pattern of gene expression ${ }^{13}$. However, in malignant conditions such as AML, p16INK4A gene expression is reduced with aging, which is mainly due to hyper-methylation of the CDKN2A promoter ${ }^{14}$. Sub-clinical studies have shown that some demethylase agents, such as cladribine and clofarabine, en- 
hance the cytotoxic effect of routine AML therapies by epigenetic modulation of TSGs, such as CDKN2A ${ }^{15}$. We also evaluated the gene expression of UHRF1, an oncogene which suppresses the expression of TSGs (such as CDKN2A) in solid tumors ${ }^{16}$. UHRF1 is responsible for histone modification (by interacting with G9a, HDAC1 and Suv39H1 proteins) and DNA methylation (by interacting with DNA methyltransferases (DNMTs) to condense the genome and reduce its accessibility to transcription factors ${ }^{16}$. On the other hand, UHRF1 marks DNMT1a/3 for proteosomal degradation and, thereby, causes genomewide hypo-methylation ${ }^{17}$.

Regarding genome-wide hyper-methylation ${ }^{9}$ and subsequent CDKN2A promoter hyper-methylation ${ }^{18}$ in leukemic blasts of AML, to our knowledge, this is the first study to evaluate UHRF1 gene expression level as an important epigenetic regulator and to investigate its correlation with p16INK4B gene expression. These investigations may help us to better understand the processes involved in TSG deregulation in elder AML patients.

\section{METHODS}

\section{Patients}

Peripheral blood (PB) and bone marrow (BM) samples were obtained at the time of diagnosis (prior to cytotoxic chemotherapy) during routine clinical assessment of 50 de novo AML patients. Specimens were collected from all patients with informed consent in agreement with the Declaration of Helsinki and the Ethical Committee of Shahid Beheshti University of Medical Sciences (IR.SBMU.RETECH.REC.1396.800). Diagnosis was made based on PB or BM film examination, immunophenotyping, and molecular studies. Immunophenotyping analysis was based on the World Health Organization (WHO) classification. Detailed demographic and sub-clinical characteristics of the patient samples are summarized in Table 1 . We also collected 18 control samples from PB and BM of healthy persons.

\section{RNA extraction and CDNA synthesis}

Mononuclear cells were isolated from PB or BM samples using Ficoll-Hypaque (INTRON, South Korea) density gradient centrifugation. These specimens were immediately cryopreserved or prepared for RNA extraction. Total RNA was extracted from each specimen using $1 \mathrm{ml}$ of Trizol. The quantity and quality of total RNA and its contamination with genomic DNA were examined by Nanodrop (Thermo Scientific, USA) and agarose gel electrophoresis. RNA to
cDNA conversion was performed according to the cDNA kit (from Thermo Scientific).

\section{Analysis of gene expression by Real-time quantitative PCR (RT-qPCR)}

A SYBR Green I Real-time PCR assay was performed in $25 \mu \mathrm{l}$ final reaction volume using $5 \mu \mathrm{l}$ cDNA (100 ng RNA equivalent), $0.75 \mu \mathrm{l}$ primers (300 nM), 12.5 Universal Master Mix (Ampliqon, Denmark), $2.5 \mu \mathrm{l}$ PCR buffer $10 \mathrm{X}$, and sterile $\mathrm{dH} 2 \mathrm{O}$ to reach total volume. Thermal cycling was carried out on Rotor-Gene 6000 (Qiagen, USA) using the following cycling conditions: $10 \mathrm{~min}$ at $95^{\circ} \mathrm{C}$, followed by 40 cycles at $95^{\circ} \mathrm{C}$ for $15 \mathrm{~s}$, and $60^{\circ} \mathrm{C}$ for $30 \mathrm{~s}$. Efficiency of all primers was evaluated by triplicate testing of five serial dilutions of cDNA. The fold changes of each gene were calculated by $\Delta \Delta$ CT formula ${ }^{19}$.

\section{Statistical analyses}

Statistical data were expressed as mean \pm SD. All tests were performed in triplicates and standard deviation (SD) less than 0.167 was admissible as a good interrun reproducibility. Depending on the Shapiro-Wilk test results, we used One-Way ANOVA or KruskalWallis for multi-state variables, and t-test or MannWhitney U for two-state variables. For analysis of correlation, Spearman test was used. Two tailed P-value less than 0.05 was considered as significant (Table 2).

\section{RESULTS}

Overall, 50 AML patients and 18 healthy volunteers were evaluated in this study. The age of the patients in the study was between $2-89$ years (median 47 years). Patients were divided in three distinct groups, including AML M0-M2 ( $\mathrm{n}=26,52 \%)$, AML M3 (2=18, 36\%), and AML M4-M5 ( $n=6,12 \%)$. Based on immunophenotyping parameters (e.g. gate of CD45 dim, the samples ranged from $20-96 \%$ (median $80 \%$ ) blast. The control group included 5 males and 13 females, aged 13-87 years (median 35 years) (Table 1 ).

As depicted in Figure 1A, p16INK4A showed increased expression in 13 (26\%) of the patients, intermediate expression in $14(28 \%)$ of patients, and a reduced expression in 23 (46\%) of patients. In search for criteria that could impact $p 16 I N K 4 A$ gene expression, we compared gene expression in different sub-groups based on age, gender, blast percent, CD34, HLA-DR positive or negative, and $\mathrm{FAB}$ sub-types. We also evaluated the correlation of $p 16 I N K 4 A$ with continuous variables, including age and blast percent. When comparing any group, no statistically significant difference was observed, except for a negative significant 
Table 1: Summary of patient's demographic data

$\begin{array}{ll}\text { Study population }(\mathbf{N}=\mathbf{5 0}) & \\ \text { Age median, y (Range, y) } & 35(3-89) \\ \text { Sex (Male/Female) } & 21 / 29 \\ \text { Sample type (Peripheral blood/Bone marrow) } & 12 / 38 \\ \text { Blast percent (Range \%) } & 80(20-98) \\ \text { Immunological Classification (\%) } & 26(52) \\ \text { AML M0-M2 } & 18(36) \\ \text { AML M3 } & 6(12)\end{array}$

AML M4, M5

Table 2: Real-time PCR oligonucleotide primers

\begin{tabular}{lllcc}
\hline \multicolumn{1}{c}{ Genes } & \multicolumn{1}{c}{ Primers } & \multicolumn{1}{c}{$\begin{array}{c}\text { Sequences } \\
\left(\mathbf{5}^{\prime}-\mathbf{3}^{\prime}\right)\end{array}$} & $\begin{array}{c}\text { TM } \\
\left({ }^{\circ} \mathbf{C}\right)\end{array}$ & $\begin{array}{c}\text { Amplicon } \\
\text { size }(\mathbf{b p})\end{array}$ \\
CDKN2A & CDKN2A.F & GCACCAGAGGCAGTAACCA & 59.63 & 101 \\
& CDKN2A.R & AGTTTCCCGAGGTTTCTCAGAG & 59.70 & \\
\multirow{2}{*}{ UHRF1 } & UHRF1.F & GCGGGGCTTCTGGTACGAC & 63 & 123 \\
& UHRF1.R & TCCACGAAGATGATCCGACAGTC & 62.04 & \\
ABL & ABLF & TGGAGATAACACTCTAAGCATAACTAAAG & 59.1 & 124 \\
& ABLR & GATGTAGTTGCTTGGGACCCA & 60.0 & \\
\hline
\end{tabular}

correlation with the age of patients (Figure 2A, r=0.034 , and $\mathrm{P}=0.016$ ). Based on the regression lines in Figure $2 \mathrm{~A}, 80.64 \%$ of patients with age above 45 years showed a decreased expression of p16INK4A, lower than the age-matched control group. However, this situation was observed only in $32 \%$ of patients below 45 years. In contrast to AML patients, p16INK4A expression had a positive significant correlation with age in the control group (Figure 2A, control group $\mathrm{r}=$ $0.50)$.

We also evaluated UHRF1 gene expression between different subgroups involved in this study. UHRF1 gene expression was down-regulated in 39 (78\%) AML patients, compared with the control group (Figure 1A, Mann-Whitney $\mathrm{U}$ test $\mathrm{P}=0.001$ ). The expression of UHRF1 gene was significantly correlated to age of both normal subjects and AML patients, but in a reciprocal manner (Figure $2 \mathbf{B}$, control group $\mathrm{r}=-0.45, \mathrm{P}=0.05$; AML patients $\mathrm{r}=0.34, \mathrm{P}=0.52$ ). Finally, we found that the gene expression of UHRF1 and $p 16 I N K 4 A$ showed a negative correlation in AML patients, but not in the control group (Figure 2C, r=0.343, $\mathrm{p}=0.015$ ).

\section{DISCUSSION}

DNA methylation pattern is commonly an agedependent process, which is characterized by a drift in CpG island epigenetic marks during aging. This drift gradually causes a distinctive pattern of epigenetic marks in old and young AML patients ${ }^{1,18,20,21}$. A study showed that the promoter of CDKN2B gene is de-methylated in healthy elder, which causes a higher expression of this gene in older individuals compared with younger ones ${ }^{20}$. This pattern of over-expression can induce apoptosis or cellular senescence in cells with genomic damage and is regarded as a protective mechanism against cancer formation ${ }^{14}$. However, cancer cells reduce the expression and also function of p16INK4A to overcome this barrier ${ }^{7}$. For example, several types of solid tumors increase the expression of UHRF1, an oncogene that recruits methylase enzymes to the CDKN2B promoter for repressing its expression ${ }^{16}$.

In contrast with solid tumors, we demonstrated that UHRF1 expression was significantly decreased in $78 \%$ of AML patients (39/50 patients). Routinely, $U H R F 1$ over-expression in solid tumors enhances tumor growth and prevents cellular differentiation and senescence ${ }^{2}$. UHRF1 overexpression in patients with breast cancer downregulates BRCA1 tumor suppressor and is associated with lower survival rate ${ }^{21}$. Various studies have also shown that UHRF1 is overexpressed in other solid tumors such as lung cancer $^{22}$, liver cancer ${ }^{23,24}$, gastric cancer ${ }^{25}$, colorectal cancer $^{26,27}$, and prostate cancer ${ }^{28}$. It impacts clinical stage, metastasis, progression, relapse of disease, and overall survival of patients. Compelling evidence have 


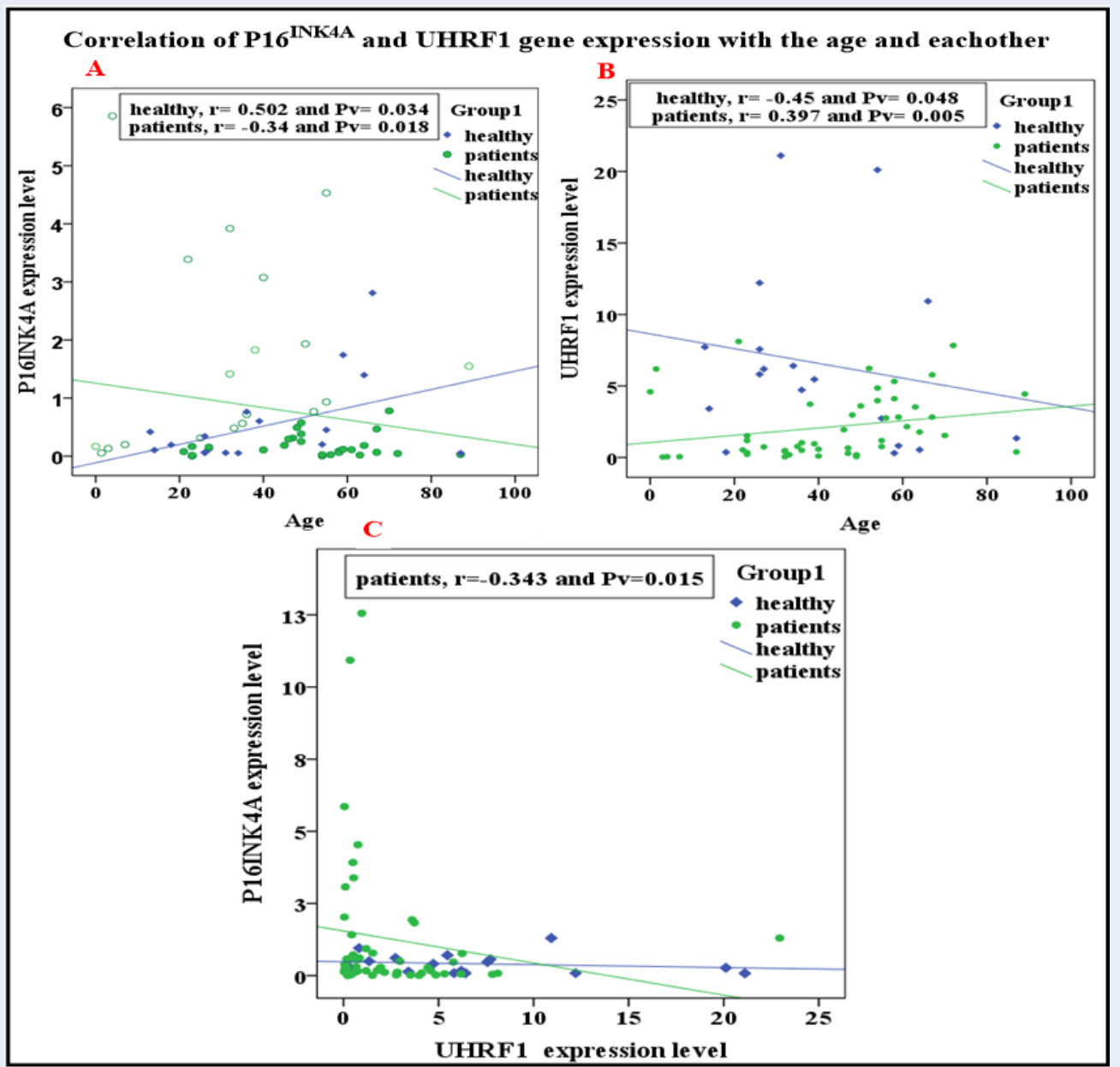

Figure 1: Correlation of P16INK4A and UHRF1 at the level of gene expression and also with the age of healthy and leukemic subjects. Green fitted lines to dots represents the regression of patients and blue lines are for healthy subjects. A and B: Spearman correlation rank was significant between age and the gene expression levels of both P16INK4A and UHRF1 when data split by healthy and patient's subgroups. As depicted in figure $\mathrm{A}$, When P16INK4A gene expression was adjusted by the age, $>80 \%$ of patients older than 45 years old express this gene more than healthy subjects but this situation was occurred in only $32 \%$ of patients younger than 45 years old. We also found a significant correlation between P16INK4A and UHRF1 gene expression in patients but not healthy subjects.

revealed that although UHRF1 over-expression induces global DNA hypo-methylation through DNMT degradation ${ }^{17}$, it simultaneously recruits methylase to the genomic regions of TSGs, such as CDH1, P16, P53, P21, KISS1 and PML, and suppresses their expression in malignant conditions ${ }^{16}$.

Based on these observations, if over-expression of UHRF1 is useful for malignant cells, it is thereby unclear as to why AML leukemic blasts downregulate UHRF-1 expression. A previous report by Mizuno et al. showed that DNMTs increased in AML patients in comparison with the bone marrow normal cells ${ }^{19}$. Further studies revealed that, DNMT enzymes are marked by UHRF1 for future degradation by proteasome system ${ }^{17}$. Therefore, regarding our data, UHRF1 down-regulation can be a possible mechanism, underlying DNMT overexpression in AML patients, which is consistent with genomic hypermethylation that occurs in many TSG regions of AML blasts $^{9}$. Additionally, other studies on human hepatocellular carcinoma cell lines have demonstrated that UHRF1 deficiency led to an expansion of cancer cells by CXCR4/AKT-JNK/IL-6 signaling pathway activation $^{29}$.

Furthermore, we also detected a significant positive correlation between the age of AML patients and 


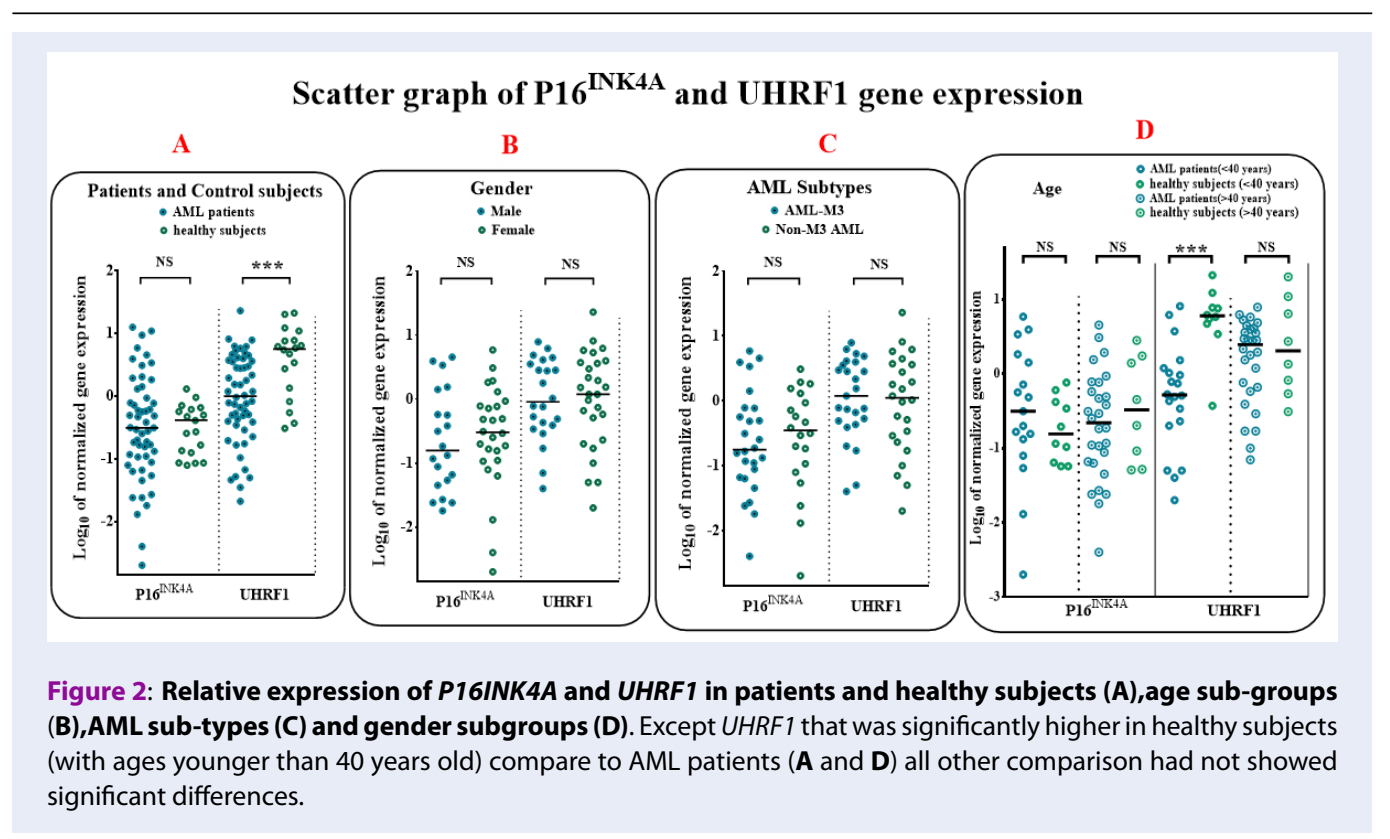

UHRF1 gene expression levels $(r=0.397$ and $\mathrm{p}=0.005)$. In our study, patients above 50 years old had a tendency to express UHRF1 in a similar method with healthy subjects, while younger patients had lower levels of UHRF1 in comparison with healthy counterparts. Thus far, no study before ours has investigated UHRF1 gene expression and its correlation with aging.

Consistent with the results obtained from solid tumors ${ }^{16}$, in the present study, UHRF1 gene expression had a significant negative correlation with $p 16 I N K 4 \mathrm{~A}$ gene expression. Evidence from the evaluation of various types of human cancers, including gastric cancer, colorectal cancer, lung cancer, cervical cancer and pancreatic carcinoma, showed that UHRF1 caused suppression of p16INK4A gene expression through hyper-methylation of its genomic locus ${ }^{16}$.

We found that more than $80 \%$ of patients who were older than 45 years expressed p16INK4A at a lower level than the normal control group. However, this situation was seen in only $32 \%$ of patients below 45 years (on the other hand, $68 \%$ of patients below 45 years had p16INK4A over-expression). p16INK4A over-expression in younger patients can be a consequence of their positive regulator over-expression ${ }^{30}$ or a physiological response to keep cells from leukemogenesis lesions, as well as preventing occurrence during aging in normal people ${ }^{14}$. However, this physiological barrier has some essential defects in elderly AML patients. Consistent with our observations, de Jonge et al. reported that p16INK4A gene expression is reduced by aging in AML patients and impacts overall survival of old patients ${ }^{9,31}$ looking for a reason. We found that old patients who had downregulation of $p 16 I N K 4 A$, express UHRF1 similar to their healthy counterpart. We suggest that a regulated pattern of $U H R F 1$ gene expression is needed to reduce p16INK4A gene expression in AML patients. This regulated pattern not only prevents over-degradation of DNMT enzymes that generally occur in solid tumors (probably due to UHRF1 over-expression) but it can also properly recruit DNMTs to the promoter of $C D K N 2 A$ gene to suppress its expression.

\section{CONCLUSIONS}

In this study, for the first time, UHRF1 has been shown to act as a repressor of p16INK4A in elderly AML patients. Down-regulation of p16INK4A may suppress cell physiological defenses against leukemogenesis and dangerous lesions, and may facilitate the development of AML in elderly people.

\section{COMPETING INTERESTS}

The authors declare no conflict of interest.

\section{AUTHORS' CONTRIBUTIONS}

All authors contributed to the design of the research. VA, MM, MKHF and AGH collected the data. VA, MAF, AH and MM conducted analysis and interpretation of data. All authors drafted the first version. VA, MAF, MM, MKHF, AGH, HA and ZKH edited the first draft. All authors reviewed, commented and approved the final draft. 


\section{ACKNOWLEDGMENTS}

Financial support: Shahid Beheshti University of Medical Sciences, Shahid Beheshti, Iran.

\section{ABBREVIATIONS}

AML: Acute Myelogenous Leukemia

PCR: Polymerase Chain Reaction

\section{REFERENCES}

1. Khwaja A, Bjorkholm M, Gale RE, Levine $R L$, Jordan $C T$, Ehninger G. Acute myeloid leukaemia. Nature Reviews Disease Primers. 2016;2:16010. Available from: DOI:10.1038/ nrdp.2016.10.

2. Saultz JN, Garzon R. Acute Myeloid Leukemia: A Concise Review. Journal of Clinical Medicine. 2016;5:E33. Available from: DOI:10.3390/jcm5030033.

3. Network CGAR. Genomic and Epigenomic Landscapes of Adult De Novo Acute Myeloid Leukemia. The New England Journal of Medicine. 2013;p. 2059-74. Available from: DOI: 10.1056/NEJMoa1301689.

4. Tsai CT, So CW. Epigenetic therapies by targeting aberrant histone methylome in AML: molecular mechanisms, current preclinical and clinical development. Oncogene. 2017;36:1753-9. Available from: DOI:10.1038/onc.2016.315.

5. Baylin SB. DNA methylation and gene silencing in cancer. Nature Clinical Practice Oncology. 2005;2:S4-11. Available from: DOI:10.1038/ncponc0354.

6. Li S, Garrett-Bakelman FE, Chung SS, Sanders MA, Hricik T, Rapaport F. Distinct evolution and dynamics of epigenetic and genetic heterogeneity in acute myeloid leukemia. Nature Medicine. 2016;22:792-9. Available from: DOI:10.1038/ nm.4125.

7. Alexandrov LB, Nik-Zainal S, Wedge DC, Aparicio SA, Behjati $\mathrm{S}$, Biankin AV, et al. Signatures of mutational processes in human cancer. Nature. 2013;500:415-21. Available from: DOI:10.1038/nature12477.

8. Patel JP, Gönen M, Figueroa ME, Fernandez H, Sun Z, Racevskis J. Prognostic relevance of integrated genetic profiling in acute myeloid leukemia. The New England Journal of Medicine. 2012;366:1079-89. Available from: DOI:10.1056/ NEJMoa1112304.

9. Spencer DH, Russler-Germain DA, Ketkar S, Helton NM, Lamprecht TL, Fulton RS, et al. CpG island hypermethylation mediated by DNMT3A is a consequence of AML progression. Cell. 2017;168:801-816. e13.

10. Hoffbrand V, Higgs DR, Keeling DM, Mehta AB. Postgraduate haematology; 2016.

11. Wang $\mathrm{Y}$, Zhang J, Xiao $\mathrm{X}$, Liu H, Wang F, Li S. The identification of age-associated cancer markers by an integrative analysis of dynamic DNA methylation changes. Scientific Reports. 2016;6:22722. Available from: DOI:10.1038/srep22722.

12. Juhl-Christensen $C$, Ommen HB, Aggerholm A, Lausen B, Kjeldsen $\mathrm{E}$, Hasle $\mathrm{H}$. Genetic and epigenetic similarities and differences between childhood and adult AML. Pediatric Blood \& Cancer. 2012;58:525-31. Available from: DOI:10. $1002 /$ pbc.23397.

13. Kim WY, Sharpless NE. The regulation of INK4/ARF in cancer and aging. Cell. 2006;127:265-75. Available from: DOI:10. 1016/j.cell.2006.10.003.

14. de Jonge $H J$, de Bont ES, Valk PJ, Schuringa JJ, Kies $M$ Woolthuis CM. AML at older age: age-related gene expression profiles reveal a paradoxical down-regulation of p16INK4A mRNA with prognostic significance. Blood. 2009;114:286977. Available from: DOI:10.1182/blood-2009-03-212688.

15. Valdez BC, Li Y, Murray D, Ji J, Liu Y, Popat U. Comparison of the cytotoxicity of cladribine and clofarabine when combined with fludarabine and busulfan in AML cells: enhancement of cytotoxicity with epigenetic modulators. Experimen- tal Hematology. 2015;43:448-61.e2. Available from: DOI: 10.1016/j.exphem.2015.02.001.

16. Ashraf W, Ibrahim A, Alhosin M, Zaayter L, Ouararhni K, Papin C. The epigenetic integrator UHRF1: on the road to become a universal biomarker for cancer. Oncotarget. 2017;8:51946-62. Available from: DOI:10.18632/oncotarget.17393.

17. Jia $Y$, Li P, Fang $L$, Zhu $H, X u$ L, Cheng $H$. Negative regulation of DNMT3A de novo DNA methylation by frequently overexpressed UHRF family proteins as a mechanism for widespread DNA hypomethylation in cancer. Cell Discovery. 2016;2:16007. Available from: DOI:10.1038/celldisc.2016.7.

18. Clark SJ, Melki J. DNA methylation and gene silencing in cancer: which is the guilty party? Oncogene. 2002;21:5380-7. Available from: DOI:10.1038/sj.onc.1205598.

19. Mizuno S, Chijiwa T, Okamura T, Akashi K, Fukumaki $Y$, Niho $Y$ Expression of DNA methyltransferases DNMT1, 3A, and 3B in normal hematopoiesis and in acute and chronic myelogenous leukemia. Blood. 2001;97:1172-9. Available from: DOI:10. 1182/blood.V97.5.1172.

20. Krishnamurthy J, Torrice C, Ramsey MR, Kovalev Gl, Al-Regaiey $\mathrm{K}$, Su L. Ink4a/Arf expression is a biomarker of aging. The Journal of Clinical Investigation. 2004;114:1299-307. Available from: Doi:10.1172/jci22475.

21. Jin W, Chen L, Chen Y, Xu SG, Di GH, Yin WJ. UHRF1 is associated with epigenetic silencing of BRCA1 in sporadic breast cancer. Breast Cancer Research and Treatment. 2010;123:35973. Available from: DOI:10.1007/s10549-009-0652-2.

22. Daskalos A, Oleksiewicz U, Filia A, Nikolaidis G, Xinarianos G, Gosney JR. UHRF1-mediated tumor suppressor gene inactivation in nonsmall cell lung cancer. Cancer. 2011;117:1027-37. Available from: DOI:10.1002/cncr.25531.

23. Wu SM, Cheng WL, Liao CJ, Chi HC, Lin YH, Tseng YH. Negative modulation of the epigenetic regulator, UHRF1, by thyroid hormone receptors suppresses liver cancer cell growth International Journal of Cancer. 2015;137:37-49. Available from: DOI:10.1002/ijc.29368.

24. Mudbhary R, Hoshida Y, Chernyavskaya Y, Jacob V, Villanueva A, Fiel MI. UHRF1 overexpression drives DNA hypomethylation and hepatocellular carcinoma. Cancer Cell. 2014;25:196209. Available from: DOI:10.1016/j.ccr.2014.01.003.

25. Zhou L, Zhao X, Han Y, Lu Y, Shang Y, Liu C. Regulation of UHRF1 by miR-146a/b modulates gastric cancer invasion and metastasis. The FASEB Journal. 2013;27:4929-39. Available from: DOI:10.1096/fj.13-233387.

26. Wang F, Yang $Y Z$, Shi CZ, Zhang P, Moyer MP, Zhang HZ. UHRF1 promotes cell growth and metastasis through repression of p16(inka) in colorectal cancer. Annals of Surgical Oncology. 2012;19:2753-62. Available from: DOI:10.1245/s10434-0112194-1.

27. Sabatino L, Fucci A, Pancione M, Carafa V, Nebbioso A, Pistore C. UHRF1 coordinates peroxisome proliferator activated receptor gamma (PPARG) epigenetic silencing and mediates colorectal cancer progression. Oncogene. 2012;31:5061-72. Available from: DOI:10.1038/onc.2012.3.

28. Babbio F, Pistore C, Curti L, Castiglioni I, Kunderfranco P, Brino L. The SRA protein UHRF1 promotes epigenetic crosstalks and is involved in prostate cancer progression. Oncogene. 2012;31:4878-87. Available from: DOI:10.1038/onc.2011.641.

29. Kim JH, Shim JW, Eum DY, Kim SD, Choi SH, Yang K. Downregulation of UHRF1 increases tumor malignancy by activating the CXCR4/AKT-JNK/IL-6/Snail signaling axis in hepatocellular carcinoma cells. Scientific Reports. 2017;7:2798. Available from: DOI:10.1038/s41598-017-02935-2.

30. Müller-Tidow C, Metzelder SK, Buerger H, Packeisen J, Ganser A, Heil G. Expression of the p14ARF tumor suppressor predicts survival in acute myeloid leukemia. Leukemia. 2004;18:720-6. Available from: DOI:10.1038/sj.leu.2403296.

31. Zeighami S, Azizzadeh E, Tabatabaee HR, Adib A, Babaei AH, Ariafar A. Opium and grade of urothelial bladder cancer Journal of Nephropathology. 2018;7(2):69-73. Available from: 10.15171/jnp.2018.17. 